Neurosurg Focus 8 (4):Introduction, 2000, Click here to return to Table of Contents

\title{
Introduction to gene therapy in neurological surgery
}

\author{
Thomas C. Chen, M.D., Ph.D., And Gregory A. Helm, M.D., Ph.D.
}

Gene therapy is an exciting new discipline in which neurosurgery and neurosurgeons can have a direct impact on both patient care and emerging scientific developments. Unlike other organs, the brain is unique in that it has a blood-brain barrier, often preventing efficient systemic gene delivery to the area of interest. Therefore, not only is gene delivery required, but it will often need to be accomplished in a local and specific manner. Although brain neoplasms have been the most commonly studied application of genetic therapeutics in neurological surgery, there are many other potential applications of this technology to neurosurgical disorders, including spinal instability, neurodegenerative disease, neurogenetic diseases, central nervous system (CNS) injury, aneurysms, trauma, stroke, and epilepsy. As the field of gene therapy for the CNS develops from the preclinical setting to clinical trials to mainstream therapy, the need for safe and specific gene delivery will be increasingly apparent. Neurosurgeons are in an enviable position as there is nobody more qualified to address the issue of how a gene can be delivered to the central nervous system. Not only do we have the training to operate on the nervous system and its coverings, but we have the ability to recognize and take care of complications that may arise from these procedures. However, the neurosurgeon's role in gene therapy for the brain and spine should not be confined to gene delivery only. Instead, we also need to understand and have a role in deciding what genes could have therapeutic utility in our patients. Herein lies our challenge in gene therapy: how can we as neurosurgeons interface with our basic science colleagues to address rapidly all of the problems that are blocking the advancement of nucleic acid therapeutics into the clinical setting.

Fortunately, as this issue of Neurosurgical Focus illustrates, a number of exciting approaches are being developed by neuroscientists and neurosurgeons throughout the world. Gene therapy is the delivery of a gene to modify the phenotypic characteristics of the target cell of interest. It may be divided into two broad categories of interest: cytotoxic and restorative. In cytotoxic gene therapy, the goal is to introduce a gene that can destroy a target cell such as a glioma cell within a glioblastoma multiforme. In restorative gene therapy, the goal is to introduce a gene that provides a new or missing function to the cell. So why would gene therapy be better than the direct administration of pharmacological agents? Gene therapy has the advantage of being able to express any therapeutic protein of interest by virtually any cell in the brain, proteins that may be impossible to deliver to the CNS by oral or intravenous administration secondary to the presence of the bloodbrain barrier. In addition, proteins will be expressed intracellularly, making it possible to manipulate cellular pathways in ways not accessible by typical therapies. The direct injection of gene therapy vectors into the brain could make it possible to express genes in specific brain regions, where high gene expression is required. Alternatively, injection of the vector into the cerebrospinal fluid could lead to diffuse protein expression, both in the ependymal lining of the ventricles and in the pia-arachnoid layers overlying the surface of the brain and spinal cord. Gene therapy also has the capability of expressing therapeutic proteins in specific cells by using tissue-specific promoters. $^{32}$ Promoters are stretches of DNA located immediately before genes. Because cells express only a small portion of the genes in their genome, cells use specific promoters to express genes specific for their particular cell type. If one of these tissue-specific promoters is placed in front of the therapeutic gene in a gene therapy vector, only cells that use that promoter will produce the therapeutic protein.

\section{METHODS OF GENE DELIVERY}

Gene therapy studies involve either ex vivo transfection techniques, in which genetically modified cells are implanted into the brain, or direct gene transfer techniques, in which viral or nonviral vectors are used to insert foreign DNA directly into brain cells. Ex vivo techniques use a variety of different cell types, including fibroblasts, stem cells, astrocytes, and immature neurons as the delivery vehicle. ${ }^{13,31,37}$ The genetically modified cells subsequently secrete the therapeutic protein that induces the desired physiological response. The crucial first step in gene therapy is the introduction of the DNA sequences of interest into the appropriate target cells. There are numerous methods currently being studied for the transfer of genetic material into cells in tissue culture and in living animals. Viral techniques in which retroviruses, adenoviruses, adeno-associated viruses, and herpes viruses are used have shown great promise and can lead to fairly high transfection rates. ${ }^{8,9,17,19,27,30}$ Nonviral techniques, includ- 
ing lipofection, direct injection of $\mathrm{DNA}, \mathrm{CaPO}_{4}$ precipitation, and ligand-DNA conjugates also look promising, although the transfection rates are typically low.

Each viral or nonviral vector has unique properties that will make it useful for specific gene therapy strategies. For instance, adenoviruses, which typically only cause minor upper respiratory and gastrointestinal infections, will readily transfect brain cells with up to $8 \mathrm{~kb}$ of DNA; however, these viruses have the disadvantage of being highly immunogenic, which may lead to a marked inflammatory response at the treatment site. ${ }^{22,25}$ Adeno-associated viruses can also transfect neurons and glia quite readily and with very little toxicity; unfortunately, only relatively small genes can be inserted into cells with these vectors. $^{9}$ In addition, it is difficult to make clinical grade vectors using adeno-associated viruses. Replication-defective herpes simplex viruses have the advantage of being able to transfect neurons, which are their usual targets in humans. ${ }^{20}$ These viruses also have the capacity to produce long-term gene expression, which may be necessary in some treatment paradigms, such as promoting neuronal regeneration. Unfortunately, these viruses have the potential to be neurotoxic and may need significant modification before they can be safely administered to humans. Retroviruses can only infect cells that are dividing, and, therefore, may have limited use for direct brain gene therapy; however, they may be ideal for ex vivo gene therapy approaches. Nonviral vectors, such as liposomes, may also be useful for inserting foreign DNA for neurosurgical applications. Current formulations are limited by the fact that they can transfect less than $1 \%$ of cells at the treatment site and also have potential to be highly toxic. Recently, significant advances have been made in the construction of liposomes, which may make them the vector of choice in the near future.

\section{Intracranial Tumors}

Advances in cytotoxic gene therapy have focused on improving vector delivery, targeting of specific genes to inhibit tumor growth, and introduction of genes to enhance cytotoxicity. Two articles in this issue address the issue of improving vector delivery. Asaoka, et al., (JNS Preview 1) have addressed the issue of vector delivery by examining why different transduction efficiencies should be observed in various glioma cell lines transduced by an adenovirus vector containing the p53 tumor suppressor gene. This issue is critical as adenovirus delivery of $p 53$ gene is currently beginning phase I trials in human patients. Understanding why some tumors may be more responsive than others to the same adenovirus infection is critical to enhance the efficiency of gene transfer. Asaoka, et al., found that differential adenoviral infection of glioma cells was dependent on the expression level of the coxsackievirus and adenovirus receptor (CAR) that is found on the surface membrane of the glioma cells. However, there was no correlation between the expression levels of the integrins avb3 and avb5, which are necessary for adenoviral internalization and viral transduction. Moreover, tumor cells transfected with the p53 gene demonstrate increased CAR expression, suggesting that a positive feedback loop may exist whereby tumor cells transduced with the p53 gene will have increased CAR expression, leading to greater viral infection. Zhu, et al., have taken advantage of the fact that glioma cells and glioma endothelial cells have overexpression of fibroblast growth factor receptors compared with normal brain. They use an adenovirus containing the suicide herpes simplex virus thymidine kinase gene and attach the basic fibroblast growth factor to its membrane. The fibroblast growth factor acts as a ligand, specifically homing the adenovirus to the glioma cells and glioma endothelial cells that overexpress the fibroblast growth factor receptors. Using this strategy, they demonstrate increased expression of the luciferase marker gene and increased cellular cytotoxicity in the targeted cells. This exciting new technology may not only provide increased specificity and efficiency in adenovirus targeting of tumor cells, but may be useful as both tumor cytotoxic and antiangiogenesis therapies.

Also in this issue, Halatsch, et al., have taken advantage of the fact that glioblastoma cells overexpress mutant epidermal growth factor receptors (EGFR) to target specific genes to inhibit tumor growth. They have used a unique agent called the ribozyme to target the EGFR gene. Ribozymes are small RNA molecules that possess the dual capability of RNA sequence-specific recognition, analogous to conventional antisense molecules, and the ability to perform site-specific cleavage of RNA substrates. Using this novel strategy, they have demonstrated that retrovirus-mediated transfer of this ribozyme to glioblastoma cells not only decreased the expression of mutant $E G F R$, but also suppressed tumor growth.

The introduction of novel cytotoxic genes is also being developed to enhance tumor cell death. In this issue, Ruan, et al., have taken advantage of the fact that glioblastomas have hypoxic regions within the tumor. Hypoxia often renders the glioma cells resistant to both chemotherapy and radiation therapy, as the hypoxic cells are in a quiescent state. This difficult state is rendered advantageous by Ruan, et al., who have coupled the bax proapoptotic gene to hypoxia responsive elements. This novel strategy allows transcriptional activation of the bax gene under hypoxic conditions, allowing for specific targeting of hypoxic tumor cells. Ryken, et al., have evaluated the feasibility of targeting the fas receptor via the fas ligand in glioblastomas. The fas receptor belongs to the tumor necrosis factor receptor family and is expressed on glioblastoma cells; targeting of the fas receptor may provide an important route for additional specificity. To determine if targeting of the fas receptor will induce apoptosis in glioblastoma cells, they have overexpressed the fas receptor in a target glioma cell line and cocultured these cells with a fibroblast cell line overexpressing the fas ligand. They demonstrate that activation of the fas receptor by the fas ligand resulted in increased glioma cell cytotoxicity. This model demonstrates that the fas receptor-fas ligand system is a potentially important new mechanism for targeting glioblastomas.

This issue of Neurosurgical Focus also contains two review articles that have special relevance to gene therapy. Basilion, et al., review some of the issues faced in gene delivery to brain tumors. Barriers unique in brain tumors include the blood-brain barrier, the blood braintumor barrier, and innate immunity. These issues are addressed in this lucid review article. To move the afore- 
mentioned studies from an in vitro setting to an in vivo model, Ding, et al., have presented their experience with the creation of spontaneously occurring astrocytomas in small animal models. These spontaneous glioma models are different from the standard models that are typically created by stereotactically implanting human glioma cells into nude rodents or implanting rodent glioma cells into an immunocompetent host. The creation of these spontaneously occurring astrocytoma models not only allows for investigation of glioma pathogenesis and progression, but more accurately recapitulates the infiltrative nature of human malignant astrocytomas.

\section{SPINAL INSTABILITY}

The paraspinal expression of osteogenic growth factors by using gene therapy techniques is currently being investigated for the induction of spinal arthrodesis. Alden, et al., initially demonstrated that the direct injection of a bone morphogenetic protein-2 adenoviral vector (AdBMP-2) can induce spinal fusion in athymic nude rats. ${ }^{2}$ Leiberman, et al., and Riew, et al., subsequently demonstrated that Ad-BMP-2 delivered via ex vivo techniques can also be used to induce endochondral bone formation. ${ }^{23,33}$ Boden's group is also investigating LIM Mineralization Protein-1 (LMP-1) ex vivo gene therapy to induce osteogenesis in vivo. ${ }^{5}$ The LMP-1 apparently upregulates the expression of a variety of osteogenic growth factors, which subsequently induce bone growth in a similar fashion to the BMPs. There are a number of advantages of using BMP gene therapy techniques to induce bone formation compared with direct protein application. The BMP expression using gene therapy does not require complex biological carriers for delivery, making it possible to induce BMP production using percutaneous delivery methods. In addition, inducible and tissue-specific promoters can be used to regulate gene expression over time and to limit transgene expression to specific cell types. The injection of a cocktail of different BMP vectors may increase the rate and quality of bone formation, possibly by the formation of BMP heterodimer, several of which have been shown to be more potent than some homodimers. In the present issue of Neurosurgical Focus, Helm, et al., have shown that BMP-13 gene therapy can induce large amounts of neotendon/ligament formation, as well as smaller amounts of bone and fibrocartilage formation. This approach may prove to be useful for improving the healing of damaged soft tissues around the spine, including the ligamentum flavum and annulus fibrosis. This approach may also be the first step toward developing a gene therapy strategy for regenerating the intervertebral disc, preventing the neurological sequelae that often occur secondary to degenerative spine disease.

\section{Neurodegenerative Disease}

The neurosurgeon may also be involved in the delivery of genes to the CNS for the treatment of neurodegenerative diseases, including Parkinson's disease, Alzheimer's disease, and amyotrophic lateral sclerosis. Numerous research groups are currently studying the use of gene therapy for the treatment of animal models of Parkinson's disease. ${ }^{4,7,12,20}$ Choi-Lundberg, et al., recently demonstrated that the glial cell line-derived neurotrophic factor $(G D N F)$ gene delivered via an adenoviral vector can prevent 6-hydroxydopamine-induced dopaminergic neuronal cell death. ${ }^{10}$ Using a recombinant adeno-associated viral vector, During, et al., also demonstrated that the delivery of the tyrosine hydroxylase genes to MPTP-treated primates can increase dopamine production in the striatum. ${ }^{11}$ Tyrosine hydroxylase gene therapy has also been performed in rodent models by using adeo-associated viral vectors, lipofection techniques, or ex vivo gene therapy. Yoshimoto, et al., also showed that astrocytes genetically modified to express brain-derived neurotrophic factor (BDNF) can induce behavioral improvement in parkinsonian rats. ${ }^{41}$ The use of neurotrophic factor gene therapy has also been suggested as a potential treatment for amyotrophic lateral sclerosis by using the ciliary neurotrophic factor and GDNF genes, and for Alzheimer's disease, using nerve growth factor, BDNF, and ciliary neurotrophic factor. ${ }^{1,6,16,26,35,38}$ Although these various strategies may be useful therapeutic options for the treatment of neurodegenerative disease, as the molecular and genetic pathophysiological mechanisms for these diseases are more fully elucidated, gene therapy aimed at preventing the disease may soon be possible.

\section{NEURONAL REGENERATION}

The potential for gene therapy to improve the regeneration of the CNS after traumatic injury has not been fully evaluated. ${ }^{36}$ Several neurotrophic factor genes have been shown to be useful in preventing cell death after axotomy and the use of this approach to improve neurite elongation, and possibly growth cone guidance, is certainly an intriguing possibility. Geschuind, et al., have demonstrated that BDNF gene therapy can induce neurite outgrowth from mammalian auditory neurons. ${ }^{15}$ Gene therapy with NGF has also been shown to induce axonal growth from cholinergic neurons, and GDNF gene therapy can increase sprouting of dopaminergic axons in the striatum. It has also become clear that inhibitory proteins within the CNS, apparently present on oligodendrocytes and reactive astrocytes, may prevent axonal regeneration. This inhibitory effect is most likely receptor and second messenger mediated and may, therefore, be accessible to genetic manipulation. For example, it may be possible to decrease the expression of the inhibitory proteins receptors on axonal growth cones, leading to improved axonal growth or decrease the rate of reactive gliosis. ${ }^{3}$ It may also be possible to upregulate neurotrophic and neurotropic receptor expression in injured neurons, making the cells more responsive to growth factors present on the brain and spinal cord.

\section{INTRACRANIAL ANEURYSMS}

Currently, the most effective technique for the endovascular occlusion of an aneurysm is the intraluminal placement of Guglielmi detachable coils. Unfortunately, coil compaction and inadequate intraluminal scarring can lead to aneurysm regrowth and rupture. One approach for improving fibrosis of aneurysms after coil placement is the seeding of genetically modified fibroblasts onto the coil prior to implantation. Transduction of the fibroblasts with 
the $b F G F$ gene may improve the rate of fibroblast proliferation and endothelial cell migration across the neck of the aneurysm. Controlling gene expression with inducible promoters, such as the tetracycline responsive system, will make it possible to regulate cellular proliferation to occlude the aneurysm maximally with scar tissue, while preventing cellular overgrowth and parent artery occlusion. Kallmes, et al., have reported the successful delivery of genetically modified tissue grafts by using detachable coils in rodents. ${ }^{18}$ This group has now shown that allogeneic rabbit fibroblasts can be delivered to and improve the healing of endovascularly induced aneurysms in the rabbit. Tissue engineering using gene therapy techniques clearly has the potential to improve vastly the efficacy of currently available technology for the endovascular occlusion of aneurysms.

\section{EPILEPSY}

The treatment of epilepsy refractory to pharmacological agents remains a neurosurgical problem. The addition of gene therapy techniques, however, may decrease the need for the surgical excision of epileptogenic foci. Zhang, et al., reported a decrease in audiogenic seizures when using cholecystokinin gene therapy in rodents. ${ }^{42} \mathrm{O}^{\prime}$ Conner, et al., reported the successful transfection of human epileptogenic brain slices with a Lac Z gene by using an adenoviral system. ${ }^{29}$ Freeze, et al., also demonstrated successful gene transfer to human epileptogenic brain slices by using an adeno-associated viral vector. ${ }^{14}$ Heat shock protein gene therapy has been shown to improve neuron survival in a kainic acid rat epilepsy model by Yenari, et al. ${ }^{40}$ In an in vitro study, Robert, et al., demonstrated successful transfer of the GAD67 gene into neurons and glia, which may be useful to induce the production of gamma aminobutyric acid, an inhibitory neurotransmitter. ${ }^{34}$ Stabilizing the membrane potentials of neurons at a seizure focus clearly is an exciting application of gene therapy, but certainly requires additional laboratory work before it is ready for clinical application.

\section{CEREBRAL ISCHEMIA}

The treatment of ischemic strokes with nucleic acid therapeutics has been successfully achieved, but may be difficult to apply clinically secondary to delays between gene transfer, transcription, and translation. ${ }^{39}$ Successful protection of the cerebral cortex has been demonstrated with bcl-2, interleukin-1, and heat shock protein gene therapy, both before and after the ischemic injury. ${ }^{21,24} \mathrm{Mu}-$ honen, et al., also demonstrated successful transduction of cerebral blood vessels after subarachnoid hemorrhage, suggesting that intracisternal or endovascular vector delivery may be successful in delivering genes that may prevent and/or reverse cerebral vasospasms secondary to subarachnoid hemorrhage. ${ }^{28}$ Nitric oxide synthase delivered with an adenoviral vector has been shown to increase nitric oxide production in cerebral vessels.

This issue of Neurosurgical Focus covers a variety of potential clinical applications of gene therapy in neurosurgery and several approaches for optimizing gene deliv- ery to the CNS. The advancement of gene therapy techniques by neurosurgeons and basic neuroscientists should make it possible to develop a wide variety of novel treatments for CNS disorders.

\section{References}

1. Aebischer P, Pochon NA, Heyd B, et al: Gene therapy for amyotrophic lateral sclerosis (ALS) using a polymer encapsulated xenogenic cell line engineered to secrete hCNTF. Hum Gene Ther 7:851-860, 1996

2. Alden TD, Pittman DD, Beres EJ, et al: Percutaneous spinal fusion using bone morphogenetic potein-2 gene therapy. J Neurosurg (Spine 1) 90:109-114, 1999

3. Audouy S, Mallet J, Privat A, et al: Adenovirus-mediated suicide gene therapy in an in vitro model of reactive gliosis. Glia 25:293-303, 1999

4. Barkats M, Bilang-Bleuel A, Buc-Caron MH, et al: Adenovirus in the brain: recent advances of gene therapy for neurodegenerative diseases. Prog Neurobiol 55:333-341, 1998

5. Boden SD, Titus L, Hair G, et al: Lumbar spine fusion by local gene therapy with a cDNA encoding a novel osteoinductive protein (LMP-1). Spine 23:2486-2492, 1998

6. Bohn MC: A commentary on glial cell line-derived neurotrophic factor (GDNF). From a glial secreted molecule to gene therapy. Biochem Pharmacol 57:135-142, 1999

7. Cao L, Zheng ZC, Zhao YC, et al: Gene therapy of Parkinson disease model rat by direct injection of plasmid DNA-lipofectin complex. Hum Gene Ther 6:1497-1501, 1995

8. Carpenter DE, Stevens JG: Long-term expression of a foreign gene from a unique position in the latent herpes simplex virus genome. Human Gene Therapy 7:1447-1454, 1996

9. Chamberlin NL, Du B de Lacalle S, Saper CB: Recombinant adeno-associated virus vector: use for transgene expression and anterograde tract tracing in the CNS. Brain Res 793:169-175, 1998

10. Choi-Lundberg DL, Lin Q, Chang YN, et al: Dopaminergic neurons protected from degeneration by GDNF gene therapy [see comments]. Science 275:838-841, 1997

11. During MJ, Samulski RJ, Elsworth JD, et al: In vivo expression of therapeutic human genes for dopamine production in the caudates of MPTP-treated monkeys using an AAV vector. Gene Ther 5:820-827, 1998

12. Fan DS, Ogawa M, Fujimoto KI, et al: Behavioral recovery in 6-hydroxydopamine-lesioned rats by cotransduction of striatum with tyrosine hydroxylase and aromatic L-amino acid decarboxylase genes using two separate adeno-associated virus vectors. Hum Gene Ther 9:2527-2535, 1998

13. Fisher LJ: Neural precursor cells: applications for the study and repair of the central nervous system. Neurobiol Dis 4:1-22, 1997

14. Freese A, Kaplitt MG, O'Connor WM, et al: Direct gene transfer into human epileptogenic hippocampal tissue with an adeno-associated virus vector: implications for a gene therapy approach to epilepsy. Epilepsia 38:759-766, 1997

15. Geschwind MD, Hartnick CJ, Liu W, et al: Defective HSV-1 vector expressing BDNF in auditory ganglia elicits neurite outgrowth: model for treatment of neuron loss following cochlear degeneration. Hum Gene Ther 7:173-182, 1996

16. Gimenez y Ribotta M, Revah F, Pradier L, et al: Prevention of motoneuron death by adenovirus-mediated neurotrophic factors. J Neurosci Res 48:281-285, 1997

17. Glatzel M, Flechsig E, Navarro B, et al: Adenoviral and adenoassociated viral transfer of genes to the peripheral nervous system. Proc Nat Acad Sci U 97:442-447, 2000

18. Kallmes DF, Williams AD, Cloft HJ, et al: Platinum coil-mediated implantation of growth factor-secreting endovascular tissue grafts: an in vivo study. Radiology 207:519-523, 1998 
19. Lachmann RH, Efstathiou S: Use of herpes simplex virus type 1 for transgene expression within the nervous system. Clin Sci 96:533-541, 1999

20. Latchman DS, Coffin RS: Viral vectors in the treatment of Parkinson's disease. Movement Disord 15:9-17, 2000

21. Lawrence MS, McLaughlin JR, Sun GH, et al: Herpes simplex viral vectors expressing Bcl-2 are neuroprotective when delivered after a stroke. J Cereb Blood Flow Metab 17:740-744, 1997

22. Le Gal La Salle G, Robert JJ, Berrard S, et al: An adenovirus vector for gene transfer into neurons and glia in the brain. Science 259:988-990, 1993

23. Lieberman JR, Le LQ, Wu L, et al: Regional gene therapy with a BMP-2-producing murine stromal cell line induces heterotopic and orthotopic bone formation in rodents. J Orthop Res 16:330-339, 1998

24. Linnik MD, Zahos P, Geschwind MD, et al: Expression of bcl2 from a defective herpes simplex virus- 1 vector limits neuronal death in focal cerebral ischemia. Stroke 26:1670-1674, 1995

25. Mannes AJ, Caudle RM, O'Connell BC, et al: Adenoviral gene transfer to spinal-cord neurons: intrathecal vs. intraparenchymal administration. Brain Res 793:1-6, 1998

26. Martinez-Serrano A, Fischer W, Soderstrom S, et al: Long-term functional recovery from age-induced spatial memory impairments by nerve growth factor gene transfer to the rat basal forebrain. Proc Nat Acad Sci 93:6355-6360, 1996

27. Mitrophanous K, Yoon S, Rohll J, et al: Stable gene transfer to the nervous system using a non-primate lentiviral vector. Gene Ther 6:1808-1818, 1999

28. Muhonen MG, Ooboshi H, Welsh MJ, et al: Gene transfer to cerebral blood vessels after subarachnoid hemorrhage. Stroke 28:822-828, 1997

29. O'Connor WM, Davidson BL, Kaplitt MG, et al: Adenovirus vector-mediated gene transfer into human epileptogenic brain slices: prospects for gene therapy in epilepsy. Exp Neurol 148: 167-178, 1997

30. Peltekian E, Parrish E, Bouchard C, et al: Adenovirus-mediated gene transfer to the brain: methodological assessment. J Neurosci Meth 71:77-84, 1997
31. Pincus DW, Goodman RR, Fraser RA, et al: Neural stem and progenitor cells: a strategy for gene therapy and brain repair. Neurosurgery 42:858-867, 1998

32. Quinn JP: Neuronal-specific gene expression--the interaction of both positive and negative transcriptional regulators. Prog Neurobiol 50:363-379, 1996

33. Riew KD, Wright NM, Cheng S, et al: Induction of bone formation using a recombinant adenoviral vector carrying the human BMP-2 gene in a rabbit spinal fusion model. Calcif Tissue Internat 63:357-360, 1998

34. Robert JJ, Bouilleret V, Ridoux V, et al: Adenovirus-mediated transfer of a functional GAD gene into nerve cells: potential for the treatment of neurological diseases. Gene Therapy 4: 1237-1245, 1997

35. Rubio F, Kokaia Z, Arco A, et al: BDNF gene transfer to the mammalian brain using CNS-derived neural precursors. Gene Therapy 6:1851-1866, 1999

36. Sapolsky RM, Steinberg GK: Gene therapy using viral vectors for acute neurologic insults. Neurology 53:1922-1931, 1999

37. Trojanowski JQ, Kleppner SR, Hartley RS, et al: Transfectable and transplantable postmitotic human neurons: a potential "platform" for gene therapy of nervous system diseases. Exp Neurol 144:92-97, 1997

38. Tuszynski MH, Roberts J, Senut MC, et al: Gene therapy in the adult primate brain: intraparenchymal grafts of cells genetically modified to produce nerve growth factor prevent cholinergic neuronal degeneration. Gene Therapy 3:305-314, 1996

39. Weihl C, Macdonald RL, Stoodley M, et al: Gene therapy for cerebrovascular disease. Neurosurgery 44:239-253, 1999

40. Yenari MA, Fink SL, Sun GH, et al: Gene therapy with HSP72 is neuroprotective in rat models of stroke and epilepsy [see comments]. Ann Neurol 44:584-591, 1998

41. Yoshimoto Y, Lin Q, Collier TJ, et al: Astrocytes retrovirally transduced with BDNF elicit behavioral improvement in a rat model of Parkinson's disease. Brain Res 691:25-36, 1995

42. Zhang LX, Li XL, Smith MA, et al: Lipofectin-facilitated transfer of cholecystokinin gene corrects behavioral abnormalities of rats with audiogenic seizures. Neuroscience 77:15-22, 1997 